\section{CÓMO PUEDE CONTRIBUIR TWITTER A UNA COMUNICACIÓN POLÍTICA MÁS AVANZADA}

\author{
Miguel Moya Sánchez \\ Universidad Carlos III de Madrid \\ m.msz@telefonica.net \\ Susana Herrera Damas \\ Universidad Carlos III de Madrid \\ dherrera@hum.uc3m.es
}

\section{HOW CAN TWITTER CONTRIBUTE TO MORE ADVANCED POLITICAL COMMUNICATION?}

Cómo citar este artículo/Citation: Moya Sánchez, M. y Herrera Damas, S. (2015). "Cómo puede contribuir Twitter a una comunicación política más avanzada". Arbor, 191 (774): a257. doi: http://dx.doi.org/10.3989/arbor.2015.774n4012
Copyright: (c) 2015 CSIC. Este es un artículo de acceso abierto distribuido bajo los términos de la licencia Creative Commons Attribution-Non Commercial (by-nc) Spain 3.0.
RESUMEN: La incorporación de nuevas funcionalidades -retuits, menciones, respuestas y etiquetas- ha convertido a Twitter en un espacio preferido para el activismo político. De ser un canal concebido para actualizaciones personales ha pasado a ser un medio para la comunicación, la conversación y la concertación política. Twitter ha demostrado su capacidad para facilitar la emancipación informativa de los ciudadanos. Esto ha despertado el interés de la dirigencia política y también ha llamado la atención de la comunidad investigadora que viene destacando aquellas facetas de Twitter que lo hacen idóneo para una comunicación avanzada. A través de un recorrido bibliográfico sobre las contribuciones más destacadas respecto al potencial que ofrece Twitter para la comunicación, hemos obtenido los fundamentos para elaborar un decálogo de sus competencias y utilidades para una comunicación política horizontal que facilite la transparencia y promueva la implicación ciudadana.

PALABRAS CLAVE: Twitter; medios sociales; comunicación política; redes sociales; comunicación avanzada.
ABSTRACT: With the incorporation of innovative functionalities -retweets, mentions, replies and hashtags- Twitter has become a favourite platform for political activism. Since its initial conception as a channel for personal updates, Twitter has turned into a medium for communication and political cooperation. Twitter has facilitated the emancipation of citizens and has aroused the interest of the political leadership. It has also attracted the attention of the research community, which has highlighted those aspects that make Twitter ideal for advanced communication. Through a bibliographic review of outstanding contributions, we have obtained the basis for developing a Decalogue of its utilities for and horizontal political communication that provides transparency and promotes citizen participation.

KEYWORDS: Twitter; social networks; political communication; social networks; advanced communication. 


\section{INTRODUCCIÓN}

La comunicación es un fenómeno social que consiste en el intento de relacionarse con los demás y que atraviesa todas las actividades humanas (Wolton, 2006). La definición de la política como la "actividad por la que se adoptan y aplican las decisiones en y para la comunidad" nos delimita el ámbito de relación de la comunicación política (Canel, 2006, p. 20). Sus manifestaciones más frecuentes son la propaganda, el marketing electoral, el marketing político y la comunicación institucional.

El uso de los medios sociales $-y$, en particular, el microblogging de Twitter- ha transformado la comunicación política rutinaria. En las redes sociales han surgido nuevos modos de comunicación que incluyen la discusión ciudadana de asuntos políticos. Los mensajes, controlados por poderosos gatekeepers en los medios tradicionales, fluyen ahora libremente en un medio pasivo en el que los comunicantes son los propios ciudadanos y los mensajes están diversificados.

Una democracia representativa de ciudadanos instruidos requiere una modalidad de comunicación en la que aquellos puedan trasladar su opinión a los gobernantes como reacción a las políticas aplicadas o que pretenden implementar. Las TICs crean los espacios para una comunicación política diferente, en la que todos los actores -ciudadanos, dirigentes políticos, periodistas y otros influyentes- pueden intervenir en igualdad de condiciones. La interacción es clave en el nuevo proceso de comunicación que proponen los medios sociales.

A la participación institucional -elecciones o asociacionismo político- se suma otra modalidad que tiene lugar en ámbitos privados con el propósito de influir en los dirigentes políticos. Es una participación reconocida como derecho en la vigente Constitución de 1978 (art. 23.1) y aconsejada por un criterio práctico que dicta que la adopción de decisiones no debe partir del supuesto de que los gobiernos poseen el mejor conocimiento de cualquier situación. El conocimiento está en la sociedad y los gobiernos no tienen otra alternativa que recurrir a ese conocimiento disperso (Innerarity, 2013). Esta forma de participar permite conocer qué piensa la gente, expresado de un modo espontáneo sin el enfoque forzado de las encuestas. Las redes sociales de comunicación cibernética (RSCC) se han revelado como espacios para el intercambio democrático de información y opinión de los ciudadanos. La estructura y funcionalidad que inaugura Twitter lo convierten en un medio idóneo para facilitar la participación ciudadana en política a través de la comunicación.
Hace unos años, el profesor Víctor Sampedro decía que el balance de ventajas e inconvenientes del uso de las nuevas tecnologías en la política estaba por hacerse y que ello dependía de que las organizaciones sociales fuesen capaces de generar prácticas comunicativas que fomentaran la autonomía de sus usuarios (Sampedro, 2006). Actualmente, el uso de medios sociales como el que nos ocupa posibilita esa autonomía.

El objetivo de esta contribución es sistematizar en qué sentidos concretos Twitter puede contribuir a una comunicación política más avanzada tanto para los dirigentes políticos como para los ciudadanos. Para ello hemos realizado una exhaustiva revisión bibliográfica poniendo en relación contribuciones que desde hace años vienen apelando a la necesidad urgente de una comunicación política más directa con otros estudios más recientes que certifican la idoneidad de Twitter para este fin.

\section{USO DE TWITTER}

A finales de julio de 2012, España contaba con unos 11 millones de usuarios de Twitter según Semiocast. Ya en ese momento se revelaba como el medio más utilizado para la comunicación entre personas, superando al teléfono. Mientras que el teléfono, fijo o móvil, eran utilizados para comunicarse con un promedio de 5,84 o 8,43 personas respectivamente, los usuarios de Twitter y redes sociales se comunicaban con 28,6 y 18,6 respectivamente (Fundación Telefónica, 2013). El uso político de Twitter despertó el interés de la fundación Orange que le dedicó una investigación adhoc. Sus conclusiones más relevantes fueron (Orange, 2013):

a) Las redes sociales favorecen significativamente el contacto directo de los ciudadanos con los partidos políticos y con las instituciones.

b) Twitter es una herramienta cada vez más utilizada por todos los partidos políticos.

c) En las Comunidades Autónomas se ha generalizado el uso de los medios sociales como canales de comunicación institucional. 14 de ellas cuentan con un perfil en Twitter.

En diciembre de 2012, el 75\% de los jefes de Estado ya utilizaban Twitter, 123 máximos líderes políticos de 164 países tenían cuentas creadas en su nombre personal o de una oficina oficial del gobierno (Digitaldaya, 2013); en 2011, eran 69. En España, según mostraba la web del Congreso, a mediados de la IX legislatura -de 2008 a 2011- el 47\% de los diputados españoles 
tenía cuenta en este servicio. A finales de 2012, eran 185 (52,85\%) y, en marzo de 2014, se llegaba a los 204 diputados (58,28\%).

Estas cifras justifican un análisis del potencial que ofrece el medio para la comunicación política. Enseguida lo vemos. Antes unas breves notas sobre la singularidad de la comunicación a través de Twitter.

\section{LA COMUNICACIÓN EN TWITTER}

Twitter proporciona un servicio de mensajería que incorpora funcionalidades existentes en otros servicios de Internet tales como el correo electrónico, la mensajería instantánea, la sindicación de noticias y el blogging (O'Reilly y Milstein, 2011).

La necesidad de que los mensajes no excedan de 140 caracteres no le impide servir como un foro para la discusión política y un indicador válido del panorama que se expresa offline (Tumasjan, Sprenger, Sandnery Welpe, 2010). 140 caracteres constituyen un "estilo de escritura breve que es profundo, inteligente, certero y humorístico" (Orihuela, 2010) que adquiere pleno sentido cuando se produce en compañía de otros: de repente, las relaciones entre personas pasan a adquirir una sensación de proximidad absoluta, incluso cuando se desarrollan a miles de kilómetros de distancia (Dans, 2011). Influyentes titulares de prensa utilizan menos de 140 caracteres.

En la comunicación reticular del medio Twitter cada usuario tiene un número de seguidores que han decidido recibir los mensajes que aquel emita. Para iniciar conversaciones se utiliza el signo @ seguido de la identidad del destinatario con el que se pretende conversar o al que se responde. El signo @ añade la funcionalidad conocida como mención que consiste en enviar el mensaje a un usuario concreto con independencia de que sea o no seguidor de quien lo emite. La utilización del signo@ fue determinante para potenciar la comunicación. Twitter pasó de ser un medio para informar a un medio que facilitaba la conversación.

Los usuarios pueden compartir con sus seguidores aquellos mensajes que consideren interesantes. En ese caso los tuits pasan a ser retuits. En la práctica, los retuits son un modo de adoptar como propios los contenidos y/o de reconocimiento de su autor además de una mención indirecta a este. A veces, también se retuitea con el propósito de dejar en evidencia al autor del mensaje retuiteado. Con la retransmisión de mensajes cuyo contenido así se suscribe, se hace llegar información y opinión a quienes pueden no tener conocimiento de ella. Desde una perspectiva po- lítica, resulta de gran interés porque los retuiteadores actúan como amplificadores del emisor causando un efecto polinizador del mensaje político.

Los tuits también puede incluir etiquetas Ilamadas hashtags, señaladas con el signo \# encabezando una palabra. De ese modo se asocia el tuit a un tema identificado con esa palabra. Si consideramos a Twitter como un medio multicanal, el signo \# señalaría a un canal en ese medio. También identificaría a un tema de conversación.

Otras dos funcionalidades nativas de Twitter son el hiperenlace y el botón de enlace Twitter. Con el primero el texto dirige al lector, de un modo transparente, a un documento en Internet. El hiperenlace, que comparte la información enlazada, compensa las posibles limitaciones comunicacionales que pueda suponer un número máximo de caracteres (140) en el mensaje. El botón de enlace Twitter en una página web permite compartir el contenido de esta con los seguidores de quienes lo pulsen. Así, establece una comunicación con Twitter desde un ámbito ajeno.

\section{ESTUDIOS RELACIONADOS}

Twitter ha sido objeto de estudio con diferentes propósitos. Unos han indagado en los motivos para su uso y en el papel que juega en la comunicación informal (Java, Song, Finin y Tseng, 2007; Zhao y Rosson, 2009). Otros destacan la importancia social que está adquiriendo (Huberman, Romero y Wu, 2009) y concluyen que un factor de su éxito clave lo constituyen las gratificaciones que Twitter proporciona a sus usuarios (Chen, 2011). Hay quienes lo identifican como un instrumento de conversación (Honeycutt y Herring, 2009) y lo examinan como tal (Boyd, Golder y Lotan, 2010) y hay quien lo ve como el boca a boca electrónico por excelencia (Jansen, Zhang, Sobel y Chowdury, 2009). Otras investigaciones han tratado de determinar la influencia que pudiera tener Twitter como medio social (Cha, Haddadi, Benevenuto y Gummadi, 2010) mientras que otros estudios se han preguntado por quiénes, cómo y por qué, son influyentes en Twitter (Bakshy, Hofman, Mason y Watts, 2011a; Bakshy, Hofman, Mason y Watts, 2011b). También se han dedicado esfuerzos a su análisis como medio de información o como red social (Kwak, Lee, Park y Moon, 2010). Asimismo, y a pesar de que sea un medio tan expuesto a una falsa información, Twitter también ha despertado interés por la credibilidad de la información que se difunde a su través (Castillo, Mendoza y Poblete, 2011). Estos trabajos seleccionados nos proporcionan el fundamento empírico para nuestro enfoque de Twitter como medio para la comunicación política. 


\section{EL POTENCIAL DE TWITTER PARA LA COMUNICACIÓN POLÍTICA}

A continuación describimos aquellas competencias funcionales que hacen de Twitter un medio de comunicación adecuado para una comunicación inmediata, directa e interactiva.

\section{1) Twitter, des-intermediador de la comunicación política}

En comunicación política, los procesos de transmisión están, en la gran mayoría de los casos, a cargo de los medios de comunicación de masas. La alteración que el medio introduce en el mensaje original forma parte de lo que llamamos mediación. Como premisa, en la comunicación de masas, está generalmente asumido que los medios causan efectos significativos en los mensajes (McQuail, 2000) porque:

a) seleccionan los mensajes que se trasladarán a las audiencias

b) les proporcionan un encuadre que puede alterar el significado del mensaje original

c) interpretan al político

Una comunicación política avanzada debe ser transparente y dejar fluir al mensaje original. Es tarea que compete al medio que ha de ser pasivo e intervenir solo en el transporte, manteniéndose ajeno en el resto del proceso.

Sin embargo, son numerosos los casos en los que el medio ha jugado el papel de militante político de modo que la mediación se ha transformado en mediatización, una sustitución de personas, instituciones o actividades sociales por el medio (Schulz, 2004). El mensaje que los medios trasladan a los políticos en nombre de la sociedad, erigiéndose en intérpretes de la opinión pública, está condicionado por los intereses de los propios medios. Empresas mediáticas con intereses políticos se convierten en un poder capaz de competir con los partidos políticos (Mancini, 1995). Por otra parte, la concentración mediática reduce el universo de las opiniones y facilita la instauración de un pensamiento unidimensional en el que las ideas quedan reducidas a los términos del universo mediático (Marcuse, 1993, p. 17).

Los medios han jugado y juegan diferentes papeles, desde el de vigilante del cumplimiento de las leyes por el poder político hasta el de elector oficioso de candidatos en los procesos electorales, pasando por el de moldeador de la política y de la opinión pública (Graber, 1995). Algunos han pretendido ser medios de adoctrinamiento con la legitimidad que les otorga la libertad de prensa.
Sin embargo, en circunstancias como la actual de desconfianza en los representantes y de cuestionamiento de la representatividad de los políticos elegidos en las urnas (Touraine, 1992, p. 47; Vallespín, 2013), al sistema de democracia representativa le conviene una fuerte dosis de comunicación directa entre políticos y ciudadanos. Y esta es posible, de un modo masivo, en Internet, con medios como Twitter.

En efecto, con los medios sociales desaparece la desintermediación y también la mediatización y queda así superada la importancia del medio tan relevante para quienes creen que el medio determina el mensaje (Innis, 2008; McLuhan, 2009).

\section{2) Twitter, espacio público para la conversación política}

La evolución tecnológica ha generado medios como Twitter que constituyen un nuevo espacio donde dar publicidad a aquellos asuntos políticos que se discuten en un ámbito privado, como consecuencia de las dificultades que encuentran los ciudadanos para acceder a los medios de comunicación de masas. Ese nuevo espacio que conforman los medios de Internet ha dado lugar a "la sociedad de medios" un siglo después de "la sociedad de las masas" (Ferry, 1998, pp. 19-20). Son ahora los ciudadanos y no las masas quienes utilizan los nuevos medios.

Twitter es un espacio que reúne a ciudadanos dispuestos a discutir asuntos públicos, donde se contribuye a la formación de la opinión pública. Las redes sociales de Twitter se suman a la prensa, a la radio y a la televisión como otra fuente donde se alimenta la conversación. Durante mucho tiempo la conversación sobre asuntos públicos tuvo lugar en ámbitos privados y necesitó de los medios de comunicación para salir a la luz pública. La triada mediática (prensa, radio y televisión) se hizo imprescindible en una sociedad democrática. Las redes de telecomunicación y Twitter posibilitan que se haga pública mientras está teniendo lugar sin necesidad de intermediarios y, lo que es tal vez más relevante, sin discriminación de los participantes. Actualmente, Twitter y las redes sociales están llevando a la gente corriente la posibilidad de tener una voz pública (Orihuela, 2011a).

Una investigación temprana sobre Twitter (Mischaud, 2007) mostraba que, frente al uso sugerido por sus creadores -exponer el estado personal- su principal utilización era enviar mensajes a determinadas personas, publicar pensamientos y puntos de vista personales y compartir información. Sus usuarios habían hecho de Twitter un medio para informarse de las opiniones de los conciudadanos y para conversar. 
La conversación en Twitter se halla a medio camino entre el chat y el blog. Es una conversación real, que se demuestra mediante la evidencia de actos de diálogo (Searle, 1991) basados en declaraciones, preguntas y respuestas. Investigaciones sobre la faceta conversacional en Twitter prueban que el $30 \%$ de los tuits contienen el signo @ y que 31\% de los mensajes con ese signo recibieron respuesta (Honeycutt y Herring, 2009). Para los investigadores de Microsoft hay una relación directa entre el retuit y la conversación (Boyd, Golder y Lotan, 2010). El retuiteo contribuye a una relación ecológica en la que las conversaciones están constituidas por una interacción pública de voces en un contexto de conversación compartida. Las estructuras conversacionales de Twitter no son las convencionales, por ello en vez de participar en un intercambio ordenado de interacciones, sus usuarios están presentes en varios contextos conversacionales simultáneamente. Esta situación tiene un efecto multiplicador de las conversaciones con tan solo un par de tuits (mensaje y respuesta) (Ritter, Cherry y Dolan, 2010).

Conviene al ideal democrático que existan medios transparentes que sirvan de espacios que alberguen a un público masivo y en los que se desarrolle una conversación democrática. La naturaleza de la política y de la esfera pública se ven reconstituidas por las nuevas tecnologías que hacen posible un nuevo modelo de conversación en democracia (Wyatt, Katz y Kim, 2000). Los nuevos medios sociales facilitan unos modos de relación distintos, innovadores (Fowler, 2011). Con ellos, la conversación política conoce un formato distinto, más informal pero de utilidad democrática, también de relevancia política para el dirigente. El carácter social de la participación ayuda a evitar consecuencias debilitadoras del aislamiento, promueve el capital social y forja identidades colectivas (Dahlgren, 2012; Innerarity y Champeau, 2012). El formalismo institucional que requiere Schudson (1997) para la conversación normativa puede convivir perfectamente con la conversación de orden político que tiene lugar en los medios sociales. Esta no anula ni sustituye a aquella; al contrario, fortalece la legitimidad de la representación porque la alimenta desde la base.

\section{3) Twitter, una nueva esfera pública periférica}

Habermas describe la esfera pública como el espacio institucional donde tiene lugar la formación de la voluntad política a través de un flujo continuo de ideas e información relevante. A finales del siglo XVIII y principios del XIX esa esfera pública se localizaba en lugares y medios tales como clubs, salones, cafés, periódicos, libros y panfletos donde, de distintas maneras, se manifestaban los ideales del hombre de la Ilustración.

Con las nuevas tecnologías, el concepto de esfera pública emerge de nuevo e incluye medios inexistentes cuando Habermas lo alumbró. Una nueva concepción considera a la esfera pública como un lugar de información, discusión, contestación, organización y lucha política que incluye los medios de difusión, los nuevos ciberespacios así como las interacciones cotidianas cara a cara (Kellner, 2000).

Históricamente, la opinión pública se ha manifestado como la expresión pública del pensamiento dominante de la clase burguesa sobre cuestiones de interés para la nación (Speier, 1950). Públicos, espacios, procesos comunicacionales y medios de comunicación forman parte de la esfera pública donde los individuos conversan para formar un organismo público (Habermas, 1974). Pero ¿qué públicos? En su análisis de la formación de la opinión pública, Price distingue cuatro clases de público: el público en general, el que vota, el público atento y el público activo (Price, 1994). El público atento, término que Price toma de Devine (1970), se distingue por su atención a los asuntos públicos, más que por su actividad. Representa aproximadamente, dice Price, a la mitad de los que votan. El término público activo es un pequeño grupo de público atento, que cifra en no más de un $15 \%$, cuyo compromiso en asuntos políticos incluye tanto participación política institucional como informal en discusiones públicas y debates con los demás ciudadanos. Estos miembros del público compiten en el mercado de opinión, entre el público atento en busca de seguidores y conversos para sus causas (Price, 1994). El público activo es el que aquí nos concierne, el que juega el papel de líder de opinión y de nuevo prescriptor en las redes sociales donde compite con los políticos por influir.

Ante un deterioro de la esfera pública convencional-desconfianza en los medios de comunicación y descrédito de las organizaciones políticas- surgen voces en pro de instancias de nuevas esferas públicas perfeccionadas en las que los ciudadanos se puedan encontrar e intercambiar puntos de vista (Fung, 2003). Medios sociales como Twitter proporcionan estructuras idóneas para canalizar la participación en esas esferas públicas reducidas. La Ciencia Política considera a los medios sociales una herramienta de refuerzo de los mecanismos tradicionales de comunicación política. 
La perspectiva tecnológica cree que esos medios estimulan la participación offline, pues la actividad en la red se prolonga en calle. Esta última corriente de opinión piensa que los medios sociales pueden contribuir a transformar la democracia.

El empoderamiento ciudadano en la nueva esfera pública, el desplazamiento de los mass media, la entrada en juego de nuevos grupos políticos y la movilización del electorado son constituyentes claves del nuevo ecosistema comunicacional (Fung, Gilman y Shkabatur, 2013). Para los optimistas respecto al papel de los medios sociales, la tecnología rellena la laguna de la participación de los ciudadanos en los medios. La propuesta de Enzensberger de que el acceso ciudadano a los medios de producción y de comunicación electrónicos debe servir para difundir la opinión de los más desfavorecidos (Enzensberger, 1970) nos recuerda que los inconvenientes para la democratización de la esfera pública son también materiales. Para los escépticos, los nuevos medios prolongan el populismo y la política-espectáculo, aunque advierten de que no debe subestimarse el hecho de que estas "herramientas" permiten a los electores una mayor implicación política (Dader, 2012).

El modo y los canales por donde fluyen las opiniones son relevantes para que un cuerpo de opinión pueda adquirir categoría de opinión pública digna de ser considerada por las instituciones de gobierno (Benkler, 2006). Este es un aspecto fundamental para comprender cómo los individuos, en las complejas sociedades contemporáneas, a distancia unos de otros, con diferentes disposiciones materiales, intelectuales y sociales, pueden ser ciudadanos de un mismo sistema de gobierno democrático. Benkler propone cuáles deben ser las características funcionales básicas que la esfera pública contemporánea debe satisfacer (Benkler, 2006):

a) Los ciudadanos propondrían asuntos a incluir en la agenda política que recogerían las instituciones, incluidos partidos políticos y parlamentos.

b) Los partidos políticos y gobiernos publicarían hechos políticos y cursos de acción alternativos.

c) Los ciudadanos emitirían opiniones y estados de ánimo sobre el curso de acción de los hechos publicados por los políticos.

Los recursos y funcionalidades de los que dispone Twitter lo hacen idóneo para implementar las propuestas de Benkler y, de este modo, albergar un concepto de esfera pública no elitista, donde pudiera consolidarse una cierta opinión pública.
La idea de que los medios sociales juegan un papel de apoyo al cambio social mediante el fortalecimiento de la esfera pública encuentra similitud en el papel histórico de la imprenta (Shirky, 2011). Sin embargo, persisten interpretaciones diversas sobre los medios sociales que oscilan entre un enaltecimiento de la web 2.0 (Grossman, 2006; Time, 2006) hasta quienes piensan que los medios sociales socavan las jerarquías y autoridad de los medios tradicionales (Keen, 2008). El temor de estos últimos es que prevalezcan los contenidos de aficionados, lo que supondría un embrutecimiento de la información y, en lo político, un discurso cada vez más grosero y extremista (Keen, 2007).

El potencial de los medios sociales en general y de Twitter en particular radica en su apoyo a la sociedad civil y la esfera pública. Se convierten en altavoces de una voz pública que se alza para dirigirse a los poderes públicos y exigirle rendición de cuentas. La forma más prometedora de contemplar a los medios sociales es considerarlos como medios que pueden fortalecer la sociedad civil en el largo plazo. Con una interpretación de la historia que contempla los cambios positivos en la vida democrática como consecuencia, más que como origen, del desarrollo de una esfera pública fuerte (Shirky, 2011).

\section{4) Twitter para la comunicación política interpersonal}

Hasta finales del siglo XX, la materialidad del soporte de la comunicación ha determinado el formato $y$ la narrativa del mensaje. Twitter nos redime de los condicionantes propios de los medios tradicionales con los que no es posible una comunicación interactiva interpersonal. Además permite que sea universal -a cualquier hora, entre quienes sean y estén donde estén, mientras nos desplazamos-. Es un medio sin ideología, sin mensaje propio, que determina una comunicación transparente.

Twitter es una aplicación multi-soporte que se ejecuta tanto en terminales de sobremesa como en portátiles. La ergonomía de estos soportes y su conexión inalámbrica los dotan de una ubicuidad vinculada a la de su portador. El terminal de comunicación es la extensión proteica que McLuhan veía en los medios aunque, en este caso, se trata del dispositivo comunicador. Esa personalización llega al punto de dejar que se manifieste la identidad del comunicante, lo que contribuye a la personalización de la comunicación (como lo opuesto a la masa, al hombre-masa de Ortega y Gasset). 
Precisamente, la relación interpersonal es clave en los procesos de persuasión propios de la comunicación política. Por una parte, el seguimiento -observación de las comunicaciones de otros usuarios- permite constatar opiniones, actitudes y valores políticos. Por otra, desarrolla las competencias del líder de opinión como retransmisor y reforzador de los mensajes de los medios (Katz y Lazarsfeld, 1955). Twitter le da una voz pública a los prescriptores sociales, a gente que, no siendo periodista, tiene prestigio cuando habla de determinados temas.

La conveniencia para el político de participar en las redes sociales se acrecienta por los efectos de influencia duradera que tiene la interacción en las relaciones interpersonales. Estas generan y mantienen ideas y patrones de comportamiento comunes entre los interlocutores que son reacios a modificar unilateralmente (Katz, 2006). Si se trata de persuadir a los ciudadanos, las conversaciones que se sostienen en las redes sociales adquieren una importancia capital por la fortaleza de las decisiones que puedan ser adoptadas en modo grupal.

La comunicación interpersonal en las redes facilita que la conversación política pueda tener lugar entre individuos distanciados ideológica o políticamente. Las redes sociales amplían cualitativa y cuantitativamente el debate mediante la interacción de líderes de opinión políticamente opuestos (Conover et al., 2011) algo que, en circunstancias de atonía o desapego ciudadano por la política, juzgamos saludable para la democracia y que los propios políticos deben promover.

\section{5) Twitter, constructor de agenda pública}

Regularmente, el político se ha venido informando de lo que piensa la gente a través de editoriales de prensa o mediante encuestas y elecciones. Estos son, para los políticos, los portadores principales de la opinión pública. Otro tipo de relaciones entre políticos y ciudadanos apenas sí presentan oportunidades para que aquellos puedan recabar la opinión de estos últimos. Salvo brevísimas apariciones populistas durante la campaña electoral, en la práctica, el contacto de unos y otros es inexistente. Es muy difícil que los políticos reciban el juicio ciudadano que merecen sus actuaciones. No hay espacio para el feedback. Los políticos no perciben directamente el clima de opinión. Los medios de comunicación desempeñan una función de interpretación de lo que le preocupa a los ciudadanos y la trasladan a los políticos, con el correspondiente sesgo de sus intereses editoriales.
Cuando surgen medios como Twitter, ciudadanos comunes y otros activos políticamente los utilizan para difundir sus opiniones sobre asuntos que les conciernen de modo que en las redes sociales se genera una agenda pública. Además, en Twitter tiene lugar un proceso de formación temprana de agenda pública. Las noticias de alcance suelen ser conocidas antes en las redes sociales que en los medios (Phuvipadawat y Murata, 2010; Becker, Naaman y Gravano, 2011). Ello se debe a que los medios las anuncian en Twitter al mismo tiempo que las publican en sus ediciones digitales y la mayor velocidad de difusión en Twitter da lugar a que las redes sociales comiencen a discutir antes los asuntos. En otras ocasiones, las fuentes son usuarios que divulgan la primicia en Twitter. Este fenómeno de la instantaneidad informativa de Twitter contribuye a que en este medio se desarrolle un clima de opinión previo al que pueda tener lugar como consecuencia de la publicación de la noticia en los medios tradicionales. Y además, sin el tradicional encuadre de estos. Así, en las redes sociales se conforma una agenda pública antes de que la triada mediática ejerza su plena influencia con la noticia elaborada.

El proceso de formación de un clima de opinión requiere comunicación de los individuos en persona. Lógicamente es más rápido en sitios como Twitter que permiten un contacto interpersonal a cualquier hora. Como consecuencia, si bien Twitter se alimenta -aunque no exclusivamente- de los medios de comunicación, construye su propia agenda de asuntos de interés, con antelación e independencia de la prioridad que estos atribuyan a tales asuntos.

En Twitter, un operador de la agenda de asuntos de interés es el trending topic. Aunque su permanencia es extremadamente volátil revela los asuntos que han adquirido un interés creciente en Twitter. Los trending topics constituyen un mecanismo interior y propio de Twitter para formar una agenda propia de las redes sociales de Twitter. Otro operador de establecimiento de agenda es el retuit, más efectivo que el trending topic. Se ha observado que los temas que marcan tendencia en Twitter lo han hecho sobre todo por medio de la propagación a través de la red, y el retuit es la forma principal de propagar la información en Twitter. El 31\% de los tuits de trending topics son retuits y el tipo de retuit más frecuente es el que contiene noticias de fuentes de medios tradicionales (Asur et al., 2011).

Tenemos pues en Twitter mecanismos que operan para generar autónomamente tendencias que, en definitiva, reflejan los asuntos de mayor interés en las 
redes. En la medida en la que los medios incorporen a su agenda tales asuntos se producirá un fenómeno de transferencia inversa de agenda-setting (Vargo, 2011). En pro de esta migración hablan las investigaciones que revelan que los contenidos generados por los usuarios son reales y auténticos (Wahl-Jorgensen, Williams y Wardle, 2010) o que ha habido asuntos que han llamado la atención de los medios a partir de su discusión en blogs (Lewis, 2010).

En definitiva, la conversación pública en las redes trata de asuntos tematizados por los ciudadanos, no necesariamente procedentes de la agenda mediática, pues ya no son los grandes medios los únicos que determinan la agenda pública sino que la gente habla de lo que le interesa (Orihuela, 2011b). De hecho, los propios medios ponen la agenda mediática a disposición de las redes sociales que pueden alterarla al definir ellas qué es lo más importante (Noguera, 2010). Cuando los medios y los políticos se hacen eco de lo que se dice en las redes sociales hay que admitir que estas constituyen un sector de la sociedad impreciso, pero influente.

\section{6) Twitter, fuente de información y opinión diversificadas}

Desde la perspectiva política, una comunicación eficiente requiere que sea interactiva y que se pueda llevar a cabo entre comunicantes ideológicamente diversos. Twitter satisface ambas condiciones. En este servicio, la concurrencia de comunicantes de diferente ideología se ve facilitada por la accesibilidad total al medio y por la libertad para opinar públicamente sin ser, por ello, condicionado por la línea editorial. Corresponde al político seleccionar, pues el medio facilita cualquier opción y no condiciona. El escenario de la comunicación política donde se ubican los usuarios de Twitter muestra en un plano de igualdad a los tres actores principales: periodistas, políticos y ciudadanos. La diada políticos-ciudadanos es la que más nos interesa porque es la que más empodera al ciudadano. Representa una vía directa de comunicación recíproca.

En épocas en las que las instituciones y la prensa están en crisis y crece en los ciudadanos un desencanto hacia las instituciones y procedimientos democráticos (Taylor, Van de Donk y Coleman, 1999) la posición privilegiada de la prensa pierde fuerza como intermediario, debido, por una parte, al crecimiento del espacio (ilimitado) de los ciudadanos en el medio Internet $y$, de otro lado, a que aquella no puede prescindir de su línea editorial que está acompañada de un determinado sesgo político.
Los nuevos medios sociales contribuyen a la mayor libertad individual para seleccionar la información y los comunicantes. Cuanto mayor variedad de fuentes y de comunicantes, más riqueza hay en la comunicación.

En las redes sociales convergen usuarios de diferentes tendencias políticas que tratan de encontrarse con sus afines ideológicos con quienes hallar argumentos o criterios para adoptar un punto de vista determinado o tomar una decisión política. Con su estructura abierta, Twitter permite que cada comunicante decida la composición de su red de información y comunicación con lo que el político se puede asegurar una diversidad de fuentes, de información y opinión. En la medida en la que la polarización sea un problema en la democracia, medios como Twitter que faciliten la exposición a otras opiniones políticas mediante la diversificación de fuentes contribuyen a una mayor calidad democrática.

\section{7) Twitter, recurso para la involucración ciudadana}

Las redes sociales de Twitter ofrecen oportunidades para expresar ideas, quejas o propuestas políticas de los ciudadanos. ¿Se trata de un deseo extendido entre la ciudadanía o es solo una aspiración de los más activos políticamente? En 2012, el CIS publicó una investigación para comprobar las preferencias de los españoles respecto a una hipotética inhibición ciudadana en la participación política. El estudio concluyó que los españoles desean procesos políticos con una intervención ciudadana claramente superior a la existente (Navarro, Wojcieszak y Alarcón, 2012).

En sociedades democráticas el sistema debe proveer los medios materiales y los estímulos necesarios para que el deseo ciudadano de participar en política se haga realidad. La falta de recursos materiales tiene un efecto disuasorio y puede alimentar el desapego por los asuntos públicos. En España, tal como demuestra el estudio del CIS citado más arriba, hay una necesidad evidente de incrementar los cauces participativos. Incumbe al político frenar la deriva de inhibición en la política. La democracia sigilosa es la negación de la opinión pública y su ausencia deja desnortado al gobernante democrático. A los poderes públicos concierne la responsabilidad en la provisión de recursos y cuidados para el desarrollo de la opinión pública.

El ciudadano que ve que sus opiniones son oídas se sentirá más respaldado que aislado en las ideas que expresa. Si la redifusión de aquellas proviniera de un político, tal vez la gratificación del emisor sería mayor. 
Twitter proporciona mecanismos extraordinaria-mente eficaces para retransmitir los mensajes ajenos. No es solo un medio para una conversación online sino que es un medio que las personas buscan de modo activo para satisfacer la necesidad de conectar con los demás (Chen, 2011). Obtener un respaldo no solicitado de una opinión emitida o recibir una respuesta personalizada a un mensaje gratifica. Estas compensaciones inmateriales forman parte del proceso de comunicación habitual en Twitter. Sus mecanismos animan al ciudadano común a participar, a opinar, a fortalecerse en la opinión propia. Comprobar que otros comparten la opinión que se temía exponer por recelo personal a hipotéticas consecuencias de rechazo debe aumentar la autoestima y así fomentar la participación. Hay una asociación entre actividad política y autoestima de modo que los más activos presentan una mayor confianza en sí mismos (Fedi, 2001). Estos gestos comunicacionales son instrumentos a disposición del político para que pueda cumplir una función social en democracia de involucrar a los ciudadanos en la discusión de los asuntos públicos. Los medios que faciliten la comunicación contribuyen a una mayor participación y compromiso político de los ciudadanos. A medida que aumente la presencia de ciudadanos y políticos en las redes, es probable que también lo haga la discusión política de donde se puede derivar un mayor grado de compromiso político.

\section{8) Twitter, medio para la representación personal del político}

Los encuentros directos del político con los electores son infrecuentes. Sin embargo, la más efectiva de las comunicaciones es aquella que tiene lugar sin intermediación, mediante el contacto personal (Katz y Lazarsfeld, 1955). Para que tenga el efecto deseado, la presencia debe ser amigable, el político debe presentarse como alguien que escucha, alguien con rostro humano. Twitter ofrece al político una oportunidad para mejorar su reputación personal. También para dañarla si tiene algún comportamiento o desliz imprudente.

A diferencia de lo que ocurre con la comunicación partidista, en Twitter el político aparece ante sus interlocutores como alguien más próximo. Ahora, el ejercicio de persuasión le exigirá dotar de personalidad y valor a lo que diga. Para difundir su mensaje eficazmente, deberá adoptar un lenguaje actualizado, próximo al de una oralidad común. Lo que diga deberá diferenciarse del mensaje institucional e impersonal que construya su partido para dirigirse a la masa. Par- te de su éxito comunicacional residirá en la habilidad que tenga de crear una atmósfera de proximidad que anime a emitir opiniones. Este clima amigable redundará en su beneficio personal y podrá ver mejorada su imagen de persona abierta al diálogo. El desarrollo de las capacidades y competencias comunicacionales que la comunicación interpersonal exige del político agrandará su liderazgo personal, algo necesario en los sistemas de democracia representativa.

En Twitter el político expresa su afiliación abiertamente. La impronta de sus mensajes es personal, o, al menos, también personal, además de partidista o institucional. Probablemente así lo quieran sus seguidores. Una presencia responsable del político implica que pueda ser requerido para dar explicaciones de sus actos o declaraciones. $\mathrm{O}$ para rendir cuentas, algo beneficioso para la imagen de "la política" y que forma parte de la tan requerida transparencia. Además, le permite conocer de primera mano situaciones concretas de los ciudadanos, interiorizarlos, y con ello aumentar su empatía y, probablemente, generar nuevos análisis de los problemas añadiendo otros enfoques.

En ciudades de tamaño medio, donde alcalde y concejales se pueden sentir en la necesidad de responder a diario ante sus convecinos, el uso regular de Twitter es un recurso inexplorado. En 2011, en Alemania el $35 \%$ del colectivo de diputados hacía uso de Twitter para comunicarse directamente con la gente de su distrito electoral local y el $36 \%$ decía que intentaba buscar comentarios, sugerencias y nuevas ideas para su trabajo político (Stieglitz, Brockmann y Xuan, 2012).

\section{9) Twitter como instrumento de previsión electoral}

La presencia de los políticos en las redes sociales origina diversos tipos de relación con sus seguidores. También registra un amplio espectro de sentimientos, desde una fidelidad política y electoral ciegas hasta una manifiesta hostilidad política. En Twitter se replica la acogida o simpatía que los políticos tienen en el mundo offline. Bajo esta premisa, vemos conveniente emplear Twitter también para hacer previsiones o sondeos sobre preferencias electorales.

Así lo han puesto de manifiesto investigaciones recientes que certifican que es posible predecir un resultado electoral a partir del análisis de la estructura de las redes sociales de Twitter y del contenido de los mensajes, con un grado de certeza del 88\% (Pang y Lee, 2008; Liu, 2010; Livne et al., 2011). En Holanda, un grupo de investigadores de la Universidad de Groningen (Sang y Bos, 2012) concluyó en la utilidad 
del análisis de Twitter para predecir resultados electorales. Analizaron unos 65.000 mensajes publicados dos semanas antes del día de la elección y, aplicando técnicas de sentiment analysis (Liu, 2010), obtuvieron resultados muy próximos a los estimados por dos empresas especializadas en sondeos electorales. Otro estudio, realizado en España por investigadores de la Universidad Politécnica de Madrid, concluye que, en las elecciones generales de 2011, hubo una correlación entre la actividad en Twitter y los resultados electorales, aunque ello no significa que esta última determine los resultados (Borondo et al., 2012).

Investigaciones en escenarios y culturas democráticas diferentes presentan conclusiones similares. Twitter no acoge a una determinada línea de pensamiento político. La opinión de los usuarios de Twitter en las redes sociales se aproxima bastante a la de los ciudadanos, lo que habla en pro del uso de Twitter como termómetro político. Esos estudios reflejan que no hay un mundo online diferente políticamente del mundo offline, tampoco que el uso de la tecnología cambie el sentimiento político. Sí se deduce, en cambio, que la penetración de Twitter en la sociedad es tal que, estadísticamente, refleja la tendencia política de la sociedad.

\section{0) Twitter, medio idóneo para situaciones de crisis}

Mucho de lo investigado sobre el uso de medios sociales está relacionado con situaciones de crisis políticas. Las movilizaciones en Occidente contra la guerra de Irak, las que agitaron Egipto, Túnez, Libia e incluso Siria, derribando regímenes o las que siguieron al atentado del 11-M o el movimiento del 15-M son ejemplos que han dado lugar a numerosísimas investigaciones sobre el uso de los medios sociales en momentos políticos difíciles.

En todos los casos, el énfasis analítico se ha puesto en la influencia que las redes sociales ejercen tanto en los medios tradicionales como en la formación de la opinión pública. El caso del $11-\mathrm{M}^{1}$ ha sido probablemente el más tratado (López García, 2004; Varela, 2004; López-Sáez y Martínez-Rubio, 2005; Sampedro, 2005; Salaverría, 2005; Dader, 2006; Doval, 2011).

Situaciones críticas de otra naturaleza ponen de manifiesto que Twitter es un factor clave en la eficacia de la comunicación y la acción (Hughes y Palen, 2009). En el verano de 2011, barrios de Londres y otras ciudades importantes en Inglaterra sufrieron durante 5 días numerosos desórdenes públicos que se desarroIlaron con gran violencia física e incluso con pérdida de vidas humanas. 28 autoridades gubernamentales emitieron tuits orientados a prevenir rumores, suministrar información oficial, promocionar acciones legales contra los infractores y organizar las actividades post-disturbios de la comunidad. La inmediatez y el poder comunicativo del microblogging tuvieron un efecto significativo en las fases de respuesta y recuperación de situaciones de emergencia (Panagiotopoulos, Bigdeli y Sams, 2012). Otro caso ejemplar fue el del terremoto en Chile en 2010 que se empleó para examinar la fiabilidad de Twitter en tiempo de crisis.

Cuando las crisis son permanentes, por ejemplo, en casos de intervenciones militares, el papel de los medios sociales se vuelve crucial. Conseguir un conocimiento preciso del entorno y ganar la batalla de la información son objetivos estratégicos militares, por ejemplo, para los EE.UU. Y, para ello, el uso de las redes sociales se hace imprescindible (Mayfield, 2008). La capacidad y velocidad de difusión de Twitter están siendo aprovechadas por agencias oficiales estadounidenses para difundir comunicados de alerta que utilizan un argot específico.

Sin embargo, para que Twitter tenga éxito en situaciones de crisis requiere que su uso sea habitual también en situaciones de normalidad política. Llegadas aquellas, el político se podrá sentir en un entorno que familiar y sus comunicaciones no serán tomadas como las de un advenedizo oportunista.

\section{CONCLUSIONES}

Los medios de comunicación de masas tradicionales han sido instrumentos de una comunicación política dirigida a sujetos receptores. Su estructura no los dota de los recursos técnicos ni de las funcionalidades mínimas necesarias para que puedan alojar la discusión de los asuntos públicos. Por naturaleza, están diseñados para proporcionar una comunicación unidireccional. Y por principio, establecimiento y origen, su diseño está orientado a que esa comunicación sea top-down, del dirigente político al ciudadano. Además, no gozan de la confianza de una mayoría de ciudadanos ya que se les considera cooperadores del establishment político. En este contexto, la participación ciudadana en asuntos políticos ha quedado relegada al voto y al asociacionismo institucional. No obstante, otras formas de participación son legítimas y posibles.

Por su naturaleza y estructura, los medios sociales son aplicaciones informáticas que permiten estas otras formas de participación. Proporcionan un espacio para que, en sociedades instruidas y con elevada 
demografía, una opinión pública se conforme con la participación ciudadana en la discusión política. Twitter es uno de ellos, el más exitoso en cuanto a su uso por los políticos y, a diferencia de Facebook -el otro medio utilizado con frecuencia por los políticos-, facilita la comunicación de un modo interactivo y sin limitaciones de acceso previo. Esto le convierte en un medio idóneo para una comunicación política avanzada que comprende tanto la bidireccionalidad como la igualdad de oportunidades para participar.

Desde una perspectiva más concreta, la cuenta de Twitter de un político pasa a ser su medio de comunicación personal. El más ágil, rápido, eficaz y menos exigente. Las manifestaciones públicas diarias en Twitter de los diputados superan a las que la mayoría de ellos tiene en los medios convencionales. No solo eso sino que, a veces, lo que dicen los diputados en Twitter tiene el valor de la primicia porque no aparece antes en los medios. Por otra parte, la cuenta de Twitter se revela como un canal selectivo de entrada de informaciones y opiniones en un flujo continuo. Es un nuevo puesto de observación política. Además de contribuir a la formación de una imagen pública personal -al margen de la que tenga por pertenecer a un partido político- el dirigente tiene la posibilidad de diseñar su cuenta de Twitter como si se tratase de su propio medio personal de comunicación, con procedimientos rutinarios de información (envío y recogida) y atención ciudadana. Esto moderniza la comunicación personal del político que encuentra en los nuevos medios una manera de modelar por sí mismo su imagen pública a la vez que hace de ellas un personalísimo medio de comunicación. La extraordinaria capilaridad del sistema de comunicación de Twitter le proporciona además una ventaja muy apreciada por la mercadotecnia y la propaganda política: la viralidad o capacidad de difusión reticular. Adicionalmente, la desintermediación de la comunicación que ofrece la plataforma permite además al dirigente pulsar personalmente el clima de opinión de sus seguidores con respecto a su propia actuación política o sobre cualquier otro asunto.

La gratuidad de uso del servicio lo hace también atractivo para un colectivo variopinto de actores políticos. En especial para el ciudadano común que desee expresar libremente su opinión política. A este, Twitter le resulta mucho más accesible que la prensa, la radio y la televisión para aportar información, opiniones y propuestas, que son otras formas, más avanzadas, que tiene la ciudadanía de participar en política, involucrarse e incrementar su compromiso político.

Estas ventajas sumadas a otras más concretas como las posibilidades que ofrece el servicio para construir la agenda pública, hacer previsiones electorales o para difundir comunicados de alerta en situaciones de crisis configuran a Twitter como una esfera pública periférica y certifican también su enorme potencial para contribuir a una comunicación política más directa y avanzada.

\section{NOTAS}

[1] Nos referimos al ataque terrorista del 11 de marzo de 2004

\section{BIBLIOGRAFÍA}

Asur, S., Huberman, B. A., Szabo, G. y Wang, C. (2011). Trends in social media: Persistence and decay. Paper presented at the 5th International AAAl Conference on Weblogs and Social Media. Disponible en http://www.hpl.hp.com/research/ scl/papers/trends/trends_web.pdf

Bakshy, E., Hofman, J. M., Mason, W. A. y Watts, D. J. (2011a). Everyone's an influencer: quantifying influence on Twitter. En: Proceedings of the Fourth ACM International Conference on Web Search and Data Mining. New York: ACM, pp. 65-74.
Bakshy, E., Hofman, J. M., Mason, W. A. y Watts, D. J. (2011b). Identifying 'influencers' on Twitter. Paper presented at the Fourth ACM International Conference on Web Search and Data Mining, New York: ACM. Disponible en: http://dougleschan. com/the-recruitment-guru/wp-content/ uploads/2014/01/Identifying-Influencers-on-Twitter-ResearchGate.pdf

Becker, H., Naaman, M. y Gravano, L. (2011). Beyond Trending Topics: RealWorld Event Identification on Twitter. Paper presented at the International AAAl Conference on Weblogs and
Social Media. Disponible en http:// www.cs.columbia.edu/ gravano/Papers/2011/icwsm11-a.pdf

Benkler, Y. (2006). The wealth of networks: How social production transforms markets and freedom. New Haven, CT: Yale University Press.

Borondo, J., Morales, A., Losada, J. y Benito, R. (2012). Characterizing and modeling an electoral campaign in the context of Twitter: 2011 Spanish presidential election as a case study. Chaos: An Interdisciplinary Journal of Nonlinear Science, 22, 2, 023138. 
Boyd, D., Golder, S. y Lotan, G. (2010). Tweet, tweet, retweet: Conversational aspects of retweeting on Twitter. Paper presented at the System Sciences (HICSS), 2010 43rd Hawaii International Conference on. Disponible en http:// www.danah.org/papers/TweetTweetRetweet.pdf

Canel, M. J. (2006). Comunicación política. Una guía para su estudio y práctica. Madrid: Tecnos.

Castillo, C., Mendoza, M. y Poblete, B. (2011). Information credibility on Twitter. En: Proceedings of the 20th International Conference on World Wide Web. New York: ACM, pp. 675-684.

Conover, M., Ratkiewicz, J., Francisco, M., Gonçalves, B., Menczer, F. y Flammini, A. (2011). Political Polarization on Twitter. En: Proceedings of the 5th International Conference on Weblogs and Social Media. Disponible en: http://www.aaai. org/ocs/index.php/ICWSM/ICWSM11/ paper/viewFile/2847\%3Cbr/3275

Cha, M., Haddadi, H., Benevenuto, F. y Gummadi, K. P. (2010). Measuring user influence in Twitter: The million follower fallacy. Paper presented at the 4 th International AAAl Conference on Weblogs and Social Media. Disponible en http://twitter.mpi-sws.org/icwsm2010_ fallacy.pdf

Chen, G. M. (2011). Tweet this: A uses and gratifications perspective on how active Twitter use gratifies a need to connect with others. Computers in Human Behavior, 27, 2, pp. 755-762.

Dader, J. L. (2006). Cibercomunicación y pseudoperiodismo en el 11-M: $\mathrm{Pa}-$ tologías de crisis en una democracia débil. Comunicación y Pluralismo, 1, pp. 35-62.

Dader, J. L. (2012). La tranformation de la politique sur Internet: de la politique-spectacle a la cyberdemocratie d'activismme citoyen. Netcom, 26, 1-2, pp. 15-35.

Dahlgren, P. (2012). Mejorar la participación: la democracia y el cambiante entorno de la web. En: Innerarity, D. y Champagne, S. (eds.) Internet y el futuro de la democracia. Barcelona: Espasa, pp.45-67.

Dans, E. (2011). Todo va a cambiar. Tecnología y evolución. Barcelona: Deusto.

Devine, D. J. (1970). The attentive public: Polyarchical democracy. Chicago: Rand McNally.
Digitaldaya. (2013). World leaders on Twitter- Ranking Report 2013 [en línea]. Disponible en: http://www.digitaldaya. com/epetition.php?id_petition=71\#/8

Doval, M. (2011). Los medios sociales en situaciones de crisis: del $11 \mathrm{M}$ al $13 \mathrm{M}$. Vivat Academia, 117E, pp. 175-192. Disponible en: http://vivatacademia.net/ index.php/vivat/article/view/54

Enzensberger, H. M. (1970). Constituents of a Theory of the Media. New Left Review, 64, pp. 13-36.

Fedi, A. (2001). Activismo político y representación del yo. Psicología Política, 22, pp. 53-76.

Ferry, J. (1998). Las transformaciones de la publicidad política. En: Ferry, J. M., Wolton, D. et al. El nuevo espacio público. Barcelona: Gedisa.

Fowler, J. H. (2011). El poder de las redes sociales [en línea]. [Fecha de consulta: 27 de enero de 2014]. Disponible en: http://www.rtve.es/television/20110403/poder-redes-sociales/421888.shtml

Fundación Telefónica. (2013). La Sociedad de la Información en España 2012. Madrid: Ariel.

Fung, A. (2003). Survey article: recipes for public spheres: eight institutional design choices and their consequences. Journal of Political Philosophy, 11, 3, pp. 338-367.

Fung, A., Gilman, H. R. y Shkabatur, J. (2013). Six Models for the Internet Politics. International Studies Review, 15, 1, pp. 30-47.

Graber, D. (1995). Los medios de comunicación y la política americana. Los medios como gobierno en la sombra. En: Muñoz Alonso, A. y Rospir, J. I. (eds.). Comunicación Política. Madrid: Universitas.

Grossman, L. (2006). You - Yes, You Are TIME's Person of the Year [en línea]. [Fecha de consulta: 23 de enero de 2014]. Disponible en: http:// content.time.com/time/magazine/article/0,9171,1570810,00.html

Habermas, J. (1974). The public sphere: An encyclopedia article. (1964). New German Critique, 3, pp. 49-55.

Honeycutt, C., y Herring, S. C. (2009). Beyond microblogging: Conversation and collaboration via Twitter. Paper presented at the System Sciences, 2009. HICSS'09. 42nd Hawaii International
Conference on. Disponible en http:// ella.slis.indiana.edu/ herring/honeycutt.herring.2009.pdf

Huberman, B. A., Romero, D. M. y Wu, F. (2009). Social networks that matter: Twitter under the microscope. First Monday, 14, 1, p. 8.

Hughes, A. L. y Palen, L. (2009). Twitter adoption and use in mass convergence and emergency events. International Journal of Emergency Management, 6, 3, pp. 248-260.

Innerarity, D. (2013). Shifting to Intelligent societies. 2013, Global University Network for Innovation. En: Higher Education in the World 5. Knowledge. Engagement and Higher Education: Contributing to Social Change, London: Palgrave Macmillan, pp. 77-83.

Innerarity, D. y Champeau, S. (2012). Internet y el futuro de la democracia. Barcelona: Planeta.

Innis, H.A. (2008). The bias of communication. Toronto: University of Toronto Press.

Jansen, B. J., Zhang, M., Sobel, K. y Chowdury, A. (2009). Twitter power: Tweets as electronic word of mouth. Journal of the American Society for Information Science and Technology, 60, 11, pp. 2169-2188.

Java, A., Song, X., Finin, T. y Ts eng, B. (2007). Why we twitter: understanding microblogging usage and communities. En: Proceedings of the 9th WebKDD and 1st SNA-KDD 2007 Workshop on Web Mining and Social Network Analysis. New York: ACM, pp. 56-65. Disponible en http://ebiquity.umbc.edu/_file_directory_/papers/369.pdf

Katz, E. (2006). Rediscovering Gabriel Tarde. Political Communication, 23, 3, pp. 263-270.

Katz, E. y Lazarsfeld, P. F. (1955). Personal influence: The part played by people in the flow of mass communications. Glencoe, IL: Free Press.

Keen, A. (2007). Is Internet Killing Our Culture? - $A B C$ News [en línea]. [Fecha de consulta: 23 de enero de 2014]. Disponible en: http://abcnews.go.com/ WN/WebCast/story?id=3432007

Keen, A. (2008). The Cult of the Amateur: How blogs, MySpace, YouTube, and the rest of today's user-generated media are destroying our economy, our culture, and our values. London: Nicholas Brealey Publishing. 
Kellner, D. (2000). Habermas, the public sphere, and democracy: A critical intervention. En: Hahn, L. (ed.). Perspectives on Habermas. Chicago: Open Court Press, pp. 259-288.

Kwak, H., Lee, C., Park, H. y Moon, S. (2010). What is Twitter, a social network or a news media? En: Proceedings of the 19th International Conference on World Wide Web, New York: ACM, pp. 591-600. Disponible en http://www. eecs.wsu.edu/ assefaw/CptS580-06/ papers/2010-www-twitter.pdf

Lewis, S. C. (2010). Citizen journalism: motivations, methods, and momentum. En: McCombs, M.; Willard Hinsley, A. Kaufhold, K. y Lewis, S. C. The Future of News: An Agenda of Perspectives. San Diego: Cognella Print, pp. 59-76.

Liu, B. (2010). Sentiment analysis and subjectivity. En Indurkhya, N. y Damerau, F. $\mathrm{J}$ (eds.). Handbook of Natural Language Processing ( $2^{\text {nd }}$. ed.), Boca Raton, London, New York: Chapman \& Hall/CRC, pp. 627-666.

Livne, A., Simmons, M. P., Adar, E. y Adamic, L. A. (2011). The Party Is Over Here: Structure and Content in the 2010 Election. En: Proceedings of the 5th International Conference on Weblogs and Social Media. Disponible en https:// www.aaai.org/ocs/index.php/ICWSM/ ICWSM11/paper/viewFile/2852/3272

López García, G. (2004). El 11-M y el consumo de medios de comunicación. Sala de Prensa, 71. Disponible en: http://www. saladeprensa.org/art565.htm

López-Sáez, M. y Martínez-Rubio, J. L. (2005). ¿influyeron los procesos de comunicación sobre los sucesos del 11-M en las votaciones del 14-M? La percepción de los jóvenes en función de su ideología política. Revista de Psicología Social, 20, pp. 351-367.

Mancini, P. (1995). Americanización y modernización. Breve historia de la campaña electoral. En: Muñoz Alonso, A. y Rospir, J. I. (eds.). Comunicación Política. Madrid: Universitas, pp. 141-168.

Marcuse, H. (1993). El hombre unidimensional: ensayo sobre la ideología de la sociedad avanzada. Buenos Aires: Editorial Planeta-Argentina.

Mayfield, A. (2008). What is social media [en línea]. [Fecha de consulta: 23 de enero de 2014]. Disponible en: http://www.info-opseurope.com/uploadedFiles/EventRedesign/UK/2012/June/11591006/
Assets/A-Commander\%27s-Strategy-forSocial-Media---By-Thomas-D.-MayfieldIII.pdf

McLuhan, M. (2009). Comprender los medios de comunicación: las extensiones del ser humano. Buenos Aires: Paidós.

McQuail, D. (2000). Introducción a la teoría de la comunicación de masas. Barcelona: Paidós.

Mischaud, E. (2007). Twitter: Expressions of the whole self. [Tesis de máster inédita]. London School of Economics: Londres.

Navarro, C. J., Wojcieszak, M. y Alarcón, P. (2012). ¿'Democracia sigilosa' en España?: Preferencias de la ciudadanía española sobre las formas de decisión política y sus factores explicativos. Barcelona: CIS.

Noguera, J. M. (2010). Redes sociales como paradigma periodístico. Medios españoles en Facebook. Revista Latina de comunicación social, 65 [en línea]. [Fecha de consulta: 23 de enero de 2014]. Disponible en: http://www.revistalatinacs. org/10/art/891_UCAM/13_JM_Noguera.html

O'Reilly, T. y Milstein, S. (2011). The Twitter Book. New York: O’Reilly Media.

Orange, F. (2013). Informe anual 2013 sobre el desarrollo de la sociedad de la información en España [en línea]. [Fecha de consulta: 23 de enero de 2014]. Disponible en: http://www.proyectosfundacionorange.es/docs/eEspana_2013_ web.pdf

Orihuela, J. L. (2010). Slow Twitter [en línea]. [Fecha de consulta: 23 de enero de 2014]. Disponible en: http://www. ecuaderno.com/2010/03/16/slowtwitter/

Orihuela, J. L. (2011a). Twitter es comunicación pública y hay que ser prudente - Vídeo en YouTube [en línea]. [Fecha de consulta: 23 de enero de 2014]. Disponible en: https://www.youtube.com/ watch?v=Z8bwzw6ommM

Orihuela, J. L. (2011b). Mundo Twitter: una guía para comprender y dominar la plataforma que cambió la red. Barcelona: Alienta.

Panagiotopoulos, P., Bigdeli, A. Z. y Sams, S. (2012). '5 Days in August'-How London Local Authorities Used Twitter during the 2011 Riots. En Scholl, H. J. et al. (eds.) Electronic Government. Berlin, Heidelberg: Springer-Verlag, pp. 102-113.
Pang, B. y Lee, L. (2008). Opinion mining and sentiment analysis. Foundations and Trends in Information Retrieval, 2, 1-2, pp. 1-135. Disponible en: http:// www.cs.cornell.edu/home/llee/omsa/ omsa.pdf

Phuvipadawat, S. y Murata, T. (2010). Breaking news detection and tracking in Twitter. Paper presented at the Web Intelligence and Intelligent Agent Technology (WI-IAT), 2010 IEEE/WIC/ACM International Conference (vol. 3). IEEE, pp. 120-123.

Price, V. (1994). La opinión pública: esfera pública y comunicación. Barcelona: Paidós Ibérica.

Ritter, A., Cherry, C. y Dolan, B. (2010). Unsupervised modeling of Twitter conversations. Human Language Technologies: The 2010 Annual Conference of the North American Chapter of the ACL, pp. 172-180. Disponible en http://www. aclweb.org/anthology/N10-1020

Salaverría, R. (2005). Los cibermedios ante las catástrofes: del $11 \mathrm{~S}$ al $11 \mathrm{M}$. Presentado en el XIX Congreso Internacional de Comunicación. Disponible en: http://dadun.unav.edu/ bitstream/10171/5125/1/los_cibermedios_ante_las_catastrofes_del_11S_ al_11M.pdf

Sampedro, V. (ed.) (2005). 13-M. Multitudes on line. Madrid: La Catarata.

Sampedro, V. (2006). ¿Redes de nudos o vacíos? Nuevas tecnologías y tejido social. Documentación social, 140, pp. 25-38.

Sang, E. T. K. y Bos, J. (2012). Predicting the 2011 Dutch senate election results with Twitter. En: Proceedings of SANS 2012, the EACL 2012 Workshop on Semantic Analysis in Social Networks, pp. 53-60. Disponible en http://ifarm.nl/erikt/papers/sasn2012.pdf

Schudson, M. (1997). Why conversation is not the soul of democracy. Critical Studies in Media Communication, 14, 4, pp. 297-309.

Schulz, W. (2004). Reconstructing mediatization as an analytical concept. European Journal of Communication, 19, 1, pp. 87-101.

Searle, J. (1991). ¿Qué es un acto de habla? En: Valdés, L. (ed.). La búsqueda del significado. Madrid: Tecnos-Universidad de Murcia, pp. 431-448.

Shirky, C. (2011). Political Power of Social Media-Technology, the Public Sphere 
Sphere, and Political Change. Foreign Affairs, 90, pp. 28-41.

Speier, H. (1950). Historical Development of Public Opinion. American Journal of Sociology, 55, 4, pp. 376-388.

Stieglitz, S., Brockmann, T. y Xuan, L. D. (2012). Usage of Social Media for Political Communication. En: Proceedings of PACIS 2012. Paper 22.

Taylor, J., Van de Donk, W. y Coleman, S. (1999). Parliament in the Age of the Internet. Parliamentary Affairs, 52, 3, pp. 365-370.

Time (2006). Person of the Year 2006 [en línea]. [Fecha de consulta: 23 de enero de 2014]. Disponible en: http:// content.time.com/time/specials/packages/0,28757,2019341,00.html

Touraine, A. (1992). Comunicación política y crisis de la representatividad. En: Ferry, J. M. et al. El Nuevo Espacio Público. Barcelona: Gedisa, pp. 47-56.

Tumasjan, A., Sprenger, T. O., Sandner, P. G. y Welpe, I. M. (2010). Predicting elec- tions with twitter: What 140 characters reveal about political sentiment. En: Proceedings of the Fourth International AAAl Conference on Weblogs and Social Media, pp. 178-185.

Vallespín, F. (2013). Crisis de representación. [en línea]. [Fecha de consulta: 23 de enero de 2014]. Disponible en: http://politica.elpais. com/politica/2013/02/14/actualidad/1360865738_616159.html

Varela, J. (2004). El dolor y la verdad de la imagen. Sala de Prensa, 66. Disponible en: http://www.saladeprensa.org/ art546.htm

Vargo, C. (2011). Twitter As Public Salience: An Agenda-Setting Analysis. Paper presented at the Annual Meeting of the Association for Education in Journalism y Mass Communication Convention in St. Louis. Disponible en http://www.researchgate.net/profile/ Chris_Vargo2/publication/264891470_ TWITTER_AS_PUBLIC_SALIENCE_ AN AGENDA-SETTING ANALYSIS BY/ links/542964b60cf238c6- 6ea7e303f.pdf
Wahl-Jorgensen, K., Williams, A. y Wardle, C. (2010). Audience views on usergenerated content: exploring the value of news from the bottom up. Northern Lights: Film y Media Studies Yearbook, 8, 1, pp. 177-194.

Wolton, D. (2006). Salvemos la comunicación: aldea global y cultura. Una defensa de los ideales democráticos y la cohabitación mundial. Barcelona: Gedisa.

Wyatt, R. O., Katz, E. y Kim, J. (2000). Bridging the spheres: Political and personal conversation in public and private spaces. Journal of Communication, 50, 1, pp. 71-92.

Zhao, D. y Rosson, M. B. (2009). How and why people Twitter: the role that microblogging plays in informal communication at work. En: Proceedings of the ACM 2009 International Conference on Supporting Group Work, pp. 243-252. Disponible en http://www.pensivepuffin.com/dwmcphd/syllabi/info447_ au10/readings/zhao.rosson.Twitter. GROUP09.pdf 